\title{
Statine zur Primärprävention kardiovaskulärer Erkrankungen
}

\author{
Arnold von Eckardstein, \\ Walter Riesen, \\ Nicolas Rodondi, \\ Michele Genoni, \\ Urs Kaufmann, \\ François Mach
}

Im Namen der Arbeitsgruppe Lipide und Atherosklerose (AGLA), der Schweizerischen Gesellschaft für Kardiologie (SGK), und der Schweizerischen Gesellschaft für Herz- und Gefässchirurgie (SGHC)

Eine Stellungnahme des Swiss Medical Board findet sich im Anschluss auf Seite 1087

\footnotetext{
* Die Literatur findet sich im Internet unter www.saez.ch $\rightarrow$ Aktuelle Nummer oder $\rightarrow$ Archiv $\rightarrow 2014 \rightarrow 29 / 30$
}

Korrespondenz:

Prof. Dr. med. Arnold von Eckardstein

Präsident AGLA

Direktor des Instituts für

Klinische Chemie

UniversitätsSpital Zürich

Rämistrasse 100

CH-8091 Zürich

arnold.voneckardstein[at]usz.ch
Das Swiss Medical Board (SMB) hat vor kurzem einen Bericht zur Anwendung von Statinen in der Primärprävention kardiovaskulärer Erkrankungen herausgegeben [1]*. Als Experten auf dem Gebiet der Atherosklerose - einschliesslich deren Prävention - nehmen die Arbeitsgruppe Lipide und Atherosklerose (AGLA) und die Schweizerischen Gesellschaften für Kardiologie (SGK) sowie Herz- und Gefässchirurgie (SGHC) zu diesem Bericht Stellung.

\section{Vorgehen und Empfehlungen des SMB zu Sta- tinen in der kardiovaskulären Primärprävention}

Das SMB hat die erwünschten und unerwünschten medizinischen Wirkungen sowie das Kosten-Wirksamkeits-Verhältnis von Statinen in der Primärprävention, also bei Patienten ohne kardiovaskuläre Symptome oder Ereignisse in der Vorgeschichte, untersucht. Für den medizinischen Teil seines Berichtes hat das SMB laut eigenen Angaben drei Experten - über solche verfügt das SMB selber nicht - einbezogen. Inwieweit deren Informationen in den Bericht eingeflossen sind, wird nicht erwähnt und bleibt unklar. Die abschliessenden Empfehlungen des SMB lauten:

- Eine Verschreibung von Statinen zur Primärprävention soll erst in Betracht gezogen werden, wenn die anderen Möglichkeiten zur Reduktion der Risikofaktoren ausgeschöpft sind.

- Das Risiko für ein kardiovaskuläres Ereignis muss individuell gemäss den gültigen Guidelines bestimmt werden.

- Eine Verschreibung von Statinen in der Primärprävention ist bei einem Risiko für ein tödliches kardiovaskuläres Ereignis von unter 10\% in $10 \mathrm{Jah}$ ren (gemäss ESC Score) nicht indiziert.

Wir sind mit dem SMB einig, dass nicht-medikamentöse Massnahmen eine wichtige Rolle in der Primärprävention der Atherosklerose spielen und dass für die Indikation einer Statintherapie das kardiovaskuläre Risiko abgeschätzt werden soll. Wir sind hingegen entschieden gegen die Begrenzung der Statinbehandlung auf Menschen mit einem tödlichen kardiovaskulären Risiko von $>10 \%$ / 10 Jahren. Diese Anhebung der Indikationsgrenze von derzeit 5\%/10 Jahren durch das SMB ist das Ergebnis einer fehlerhaften Kosten-Effektivitäts-Analyse. Ausserdem übersieht das SMB mit seinen Empfehlungen wichtige Patientengruppen, welche laut internationaler und nationaler Empfehlungen frühzeitig mit Statinen behandelt werden müssen, nämlich Diabetiker und Patienten mit familiärer Dyslipidämie (vor allem familiäre Hypercholesterinämie), sowie Patienten mit Risikofaktoren, welche nicht im ESC Score, aber in der schweizweit am häufigsten eingesetzten Methode für die kardiovaskuläre Risikoabschätzung, dem AGLA Score, berücksichtigt sind: positive Familiengeschichte für frühzeitige kardiovaskuläre Ereignisse, niedriges HDL-Cholesterin und erhöhte Triglyzeride.

\section{Unbestrittene Wirksamkeit von Statinen in der Primärprävention}

Das SMB untersuchte 6 Meta-Analysen [2-7], die bei Patienten ohne vorbestehende kardiovaskuläre Krankheit die Wirksamkeit einer Statinbehandlung geprüft hatten. Das SMB bestätigte in seiner «Meta-MetaAnalyse» die bereits in diesen Meta-Analysen robust und konsistent abgeleitete Tatsache, dass Statine in der Primärprävention wirksam sind.

\section{Fehlerhafte Kosten-Effektivitäts-Analyse}

Das SMB berechnete die Kosteneffektivität von Statinen für die Primärprävention in der Schweiz, wobei es sich auf die Meta-Analyse der Cholesterol Treatment Trialist (CTT) Collaborators stützte. Es kam für die Subgruppe der Personen mit einem kardiovaskulären Risiko $<10 \%$ in 5 Jahren auf Kosten von rund 210000 CHF für ein gewonnenes qualitätsadjustiertes Lebensjahr (QALY). Dieses Kostenverhältnis wurde als ungünstig und im Folgenden als Hauptgrund für einen restriktiven Statin-Einsatz beurteilt. Diese Analyse hat mehrere Fehler. Die hohen QALY-Kosten hätten dem SMB schon durch Vergleich mit früheren prominent publizierten Kosten-Effektivitäts-Analysen auffallen müssen: Ein indirekter Hinweis auf die Überschätzung der Kosten pro QALY durch das SMB ergibt sich schon aus einer 2009 publizierten amerikanischen KostenEffektivitäts-Analyse zur Statintherapie in der Primärprävention [8]. Obwohl die Statine seinerzeit noch nicht generisch waren und das US-amerikanische Gesundheitssystem als noch teurer als das Schweizer gilt, wurden die Kosten pro QALY auf 201000 US-Dollar geschätzt, wenn jeder unabhängig von seinem Risiko behandelt würde. Bei einer nach ATP III geschätzten Risikoschwelle von 10\% für tödliche und nicht-tödliche Herzinfarkte in 10 Jahren (statt 5 Jahren wie beim SMB) wurden 50000 US-Dollar pro QALY als kostenef- 
fektiv angesehen. Da der ATP III Score wie der AGLA Score das Risiko für kardiovaskuläre Morbidität + Mortalität schätzt (während der ESC Score nur das Risiko für kardiovaskuläre Mortalität schätzt), muss man davon ausgehen, dass das SMB die Kosten pro QALY um mindestens Faktor 4 überschätzt.

Zwei wichtige Ursachen der Fehlberechnung durch das SMB lassen sich schon leicht durch Nachberechnung der CTT-Daten herauskristallisieren. Das SMB errechnete für die Subgruppe der CTT-Analyse mit einem Risiko von $<10 \%$ in 5 Jahren, dass die Statintherapie 11 Ereignisse pro 1000 Personen in 5 Jahren verhindert. Tatsächlich sind es aber 16,5/1000 Ereignisse in 5 Jahren. Diese Zahlen wurden von den CTT-Collaborators für eine Senkung des LDL-Cholesterins um $1 \mathrm{mmol} / \mathrm{l}$ berechnet, welche das SMB der Einfachheit halber mit der durchschnittlich erreichbaren LDL-Cholesterin-Senkung gleichsetzte. Das ist deutlich zu wenig: Bei einer durchschnittlichen LDLCholesterin-Konzentration von $3,6 \mathrm{mmol} / \mathrm{l}$ in der Schweizer Bevölkerung bedeutet $1 \mathrm{mmol} / \mathrm{l}$ eine Absenkung von weniger als 30\%. 20 mg Rosuvastatin oder $40 \mathrm{mg}$ Simvastatin senken das LDL-Cholesterin gemäss STELLAR-Trial aber um 52\% bzw. 47\% [9]. Damit wären entweder stärkere Effekte oder tiefere, günstigere Statindosierungen in die Modellrechnung des SMB einzubeziehen. Bei überdurchschnittlich hohen LDL-Cholesterin-Konzentrationen, die bevorzugt mit Statinen behandelt werden, muss man eher von $2 \mathrm{mmol} / \mathrm{l}$ LDL-Cholesterin-Senkung ausgehen.

Die Statinpreise berücksichtigen auch die in der Praxis häufige Teilung von Tabletten nicht. Die durch Statine eingesparten Kosten, d.h. verhinderte vaskuläre Ereignisse, sind nicht plausibel dargestellt. Die Kosten für vaskuläre Ereignisse werden einer Publikation über Vorhofflimmern entnommen [10], dort sind aber Revaskularisationen nicht berücksichtigt. Zudem wird erwähnt, dass das Modell die Langzeitkosten nach akutem Myokardinfarkt nicht berücksichtigt. Patienten nach vaskulären Ereignissen benötigen aber in der Regel während Jahren umfassende medizinische Betreuung. Die QALYs werden mit Hilfe einer für Tumorpatienten entwickelten Skala, des Karnofsky-Index, bestimmt; seine Validität für Kreislaufkrankheiten dokumentiert das SMB nicht. Auch wurde keine Sensitivitätsanalyse vorgelegt, welche die Robustheit der Ereignisse bestätigt hätte, wie in Publikationen mit Peer-Review üblich. Die Kosten-Effektivitäts-Analyse des SMB ist somit fehlerhaft und taugt insgesamt nicht als Entscheidungsgrundlage.

\section{Individuelle Risikoschätzung und Interventions- schwelle}

Auch als «normal» angesehene LDL-Cholesterinwerte können durch Statine gesenkt werden, wodurch das kardiovaskuläre Risiko abnimmt. Je höher aber das Risiko ist, umso kleiner wird die number needed to treat (NNT) und umso grösser wird der Nutzen, weil absolut mehr Ereignisse verhindert werden. Das SMB empfiehlt deshalb, wie die AGLA und internationale
Fachgesellschaften, den Einsatz von Statinen bei asymptomatischen Patienten vom kardiovaskulären Gesamtrisiko abhängig zu machen.

Das SMB spricht sich, ohne weitere Differenzierungen vorzunehmen, dafür aus, erst ab einem sehr hohen Risiko von 10\% für ein tödliches kardiovaskuläres Ereignis in 10 Jahren mit Statinen zu intervenieren. Dabei gilt es zu bedenken, dass neben den $>10 \%$ tödlichen Ereignissen, die dem ESC Score als Risikomass zugrunde liegen, noch doppelt so viele schwerwiegende vaskuläre Ereignisse dazu kommen [11]. Für das SMB besteht also erst dann ein Grund zur Intervention, wenn das Risiko für tödliche und nicht-tödliche vaskuläre Ereignisse in 10 Jahren mindestens $30 \%$ beträgt. Damit setzt sich das SMB über alle Hochrisikogrenzen aus internationalen und nationalen Empfehlungen hinweg, nämlich: von 5\% in 10 Jahren für tödliche kardiovaskuläre Ereignisse (Europäische Gesellschaft für Kardiologie [ESC] und European Atherosclerosis Society [EAS]), $20 \%$ in 10 Jahren für tödliche und nicht-tödliche Herzinfarkte (AGLA, Adult Treatment Panel [ATP] III, International Atherosclerosis Society [IAS]), oder 7,5\% in 10 Jahren für tödliche und nicht-tödliche atherosklerosebedingte Gefässereignisse (neue Empfehlungen des American College of Cardiologists [ACC] und American Heart Association [AHA]). Auch die CTT-Meta-Analyse, auf welche das SMB stark Bezug nimmt, empfiehlt bei einem 5-Jahres-Risiko für kardiovaskuläre Morbidität und Mortalität $>10 \% \mathrm{zu}$ behandeln (entspricht ESC ca. 7\% / 10 Jahre).

Zielwerte für LDL-Cholesterin werden im Gegensatz zu AGLA oder ESC vom SMB überhaupt nicht genannt, so dass man annehmen muss, dass der Rat des $\mathrm{SMB}$ «fire and forget» lautet. Die Bezugsquelle des SMB, die gemeinsamen Empfehlungen der ESC und EAS, empfiehlt hingegen in seiner neuesten Auflage aus dem Jahre 2011 Zielwerte für LDL-Cholesterin, die risikoabhängig avisiert werden sollen, nämlich $<1,8 \mathrm{mmol} / \mathrm{l}$ bei einem Risiko für tödliche Ereignisse $>10 \% / 10$ Jahre, $<2,5 \mathrm{mmol} / \mathrm{l}$ bei einem Risiko $<5 \% / 10$ Jahre und $<3,4$ $\mathrm{mmol} / \mathrm{l}$ bei einem Risiko von 1-5\%/ 10 Jahre.

\section{Vom SMB nicht berücksichtigte Risikofaktoren}

Das SMB macht die Indikationsstellung für Statine nur vom absoluten Risiko gemäss ESC Score abhängig. Dabei übersieht es Hochrisikogruppen, bei denen wie in der Sekundärprävention unabhängig von anderen Risikofaktoren nach allen internationalen Empfehlungen ein LDL-Cholesterin $<2,6 \mathrm{mmol} / 1$ angestrebt werden soll. Dies gilt vor allem für Diabetiker. Im Vergleich zu Nicht-Diabetikerinnen haben Frauen mit Diabetes ein 5-fach höheres und Männer mit Diabetes ein 3-fach höheres Risiko. Statine gehören zu den wenigen Medikamenten-Gruppen, die bei Diabetikern das kardiovaskuläre Risiko substantiell senken. Es ist überraschend, wenn das SMB diese wegen ihrer relativ hohen Prävalenz, ihres hohen kardiovaskulären Risikos und grossen Benefits bei Statintherapie wichtige Gruppe [12-14] bei seinen Empfehlungen zur Primärprävention völlig ausser Acht lässt. 
Schliesslich berücksichtigt der ESC Score wichtige Risikofaktoren gar nicht [11]: HDL-Cholesterin, Triglyzeride, und die Familienanamnese. Bei einem Herzinfarkt der Eltern, Grosseltern oder Geschwister vor dem 60. Lebensjahr erhöht sich das Risiko um das 1,7-Fache bei Frauen und um das 2-Fache bei Männern. Das Fehlen dieser Risikofaktoren schränkt die diagnostische Wertigkeit des ESC Scores im Vergleich zu anderen Risikobestimmungsmethoden ein. So teilt der ESC Score im Vergleich zum AGLA-Rechner viele ältere Schweizer in hohe Risikokategorien ein und überschätzt deren Risiko wahrscheinlich. Hierauf deuten die Ergebnisse einer jüngsten holländischen Validierungsstudie [15]. Die AGLA-Methode erlaubt auch durch Berücksichtigung von Familiengeschichte, HDL-C und Triglyzeriden eine sensitivere und spezifischere Identifikation von Hochrisikopersonen im jüngeren und mittleren Alter, wie für die Schweiz gezeigt wurde [16]. Tatsächlich ist der AGLA-Rechner (www. agla.ch) in der Schweiz am weitesten verbreitet und es ist unverständlich, dass das SMB dieser Tatsache nicht Rechnung trägt.

Durch die Festlegung der Risikoschwelle auf 10\% für tödliche Ereignisse in 10 Jahren begrenzt das SMB die Primärprävention durch Statine faktisch auf Personen, die 60 Jahre und älter sind. Laut ESC Score-Tabellen für Niedrigrisikoländer wie die Schweiz überschreitet eine Frau diesen Grenzwert erst, wenn sie mindestens 65 Jahre alt ist, raucht, einen Blutdruck von $160 \mathrm{~mm} \mathrm{Hg}$ und einen Cholesterin-Wert von $8 \mathrm{mmol} / \mathrm{l}$ hat. Ein nichtrauchender Mann muss ebenfalls 65 Jahre alt werden, einen Blutdruck von 160 mm Hg und eine Hypercholesterinämie von $8 \mathrm{mmol} / \mathrm{l}$ haben, um laut SMB für eine Primärprävention durch Statine in Frage zu kommen. Dem SMB folgend, müssten bei allen unter 60-Jährigen in der Schweiz die bestehenden Statintherapien in der Primärprävention gestoppt werden. Ein Abbruch der Statin-Therapie erhöht allerdings bereits kurzfristig das Risiko für kardiovaskuläre Ereignisse [17, 18].

\section{Familiäre Hypercholesterinämie}

Ein Gesamtcholesterin von 8 mmol/l entspricht der 95. Perzentile in der Schweizer Erwachsenenbevölkerung und würde in der Klinik die Verdachtsdiagnose «Familiäre Hypercholesterinämie» wecken, vor allem wenn sekundäre Ursachen wie Cholestase, nephrotisches Syndrom, Hypothyreose oder bestimmte Medikamente ausgeschlossen sind. Bei einer Gesamtprävalenz von $1 / 200$ in der Bevölkerung beträgt die Wahrscheinlichkeit bis zu 10\%, dass bei einem Gesamtcholesterin von $\geq 8 \mathrm{mmol} / \mathrm{l}$ eine familiäre Hypercholesterinämie vorliegt. Unbehandelt haben diese Patienten kaum eine Chance, das 60. Lebensjahr ohne kardiovaskuläres Ereignis zu erreichen, obwohl sie in jungen und mittleren Jahren nie ein ESC-Risiko über 10\% für tödliche Ereignisse in 10 Jahren erreichen. Deswegen sollen diese Patienten nach heutigen Empfehlungen früh behandelt werden [19], nicht aber wenn man dem SMB folgt. Damit stellt sich das SMB gegen alle Empfehlungen zum Management der familiären Hypercholesterinämie, die sogar zu einem systematischen Kaskaden-Screening in betroffenen Familien raten, um Behandlungen möglichst früh beginnen zu können.

\section{Alter}

Im Einzelfall ist auch zu prüfen, ob bei einem Menschen in mittlerem Alter und intermediärem Risiko auf eine Behandlung verzichtet werden soll, wenn absehbar ist, dass er mit 65 Jahren ein hohes Risiko erreichen wird. Tatsächlich werden heute unter der Vorstellung, dass ein Risikofaktor nicht nur durch seine Dosis, sondern auch durch die Expositionsdauer wirkt, neue RisikoAlgorithmen propagiert, bei denen 30-Jahres-Risiken (QRISK2) oder Lebensrisiken (Lloyd-Jones-Version des Framingham-Algorithmus) für kardiovaskuläre Ereignisse berechnet werden, um gezielter Präventionsmassnahmen im mittleren Lebensalter zu ergreifen.

\section{Behandlung von Risikofaktoren}

Wir stimmen mit dem SMB überein, dass das kardiovaskuläre Risiko nicht nur durch die Cholesterinsenkung vermindert werden soll, sondern alle Risikofaktoren anzugehen sind. Lebensstil-Massnahmen Ernährung, Bewegung und vor allem Rauchabstinenz - stehen am Anfang und sind bei allen Personen jeder Risikokategorie zu empfehlen. In Bezug auf die Ernährung muss allerdings festgehalten werden, dass die Evidenz für etliche Massnahmen - zum Beispiel Meidung von Cholesterin und gesättigten Fettsäuren - durch jüngste Meta-Analysen hinterfragt wurden [20, 21]. Besonders problematisch ist die Empfehlung des SMB, dass vor dem Beginn einer Statintherapie der Blutdruck korrigiert werden sollte. Für dieses schrittweise Vorgehen, dass erst bei ungenügender Risikominderung durch Blutdrucksenkung die Hypercholesterinämie behandelt werden soll, gibt es keine Studienevidenz. Entsprechend empfiehlt die AGLA die gemeinsame Behandlung aller Risikofaktoren.

\section{Navigation der cholesterinsenkenden Therapie zwischen Skylla (SMB) und Charybdis (AHA/ACC)}

Die Empfehlungen des SMB zum Statin-Einsatz, generell erst ab einem ESC-Risiko $>10 \%$ für tödliche Ereignisse zu behandeln, bergen wie oben dargelegt zahlreiche Gefahren der Unterbehandlung. Nahezu zeitgleich mit dem SMB-Bericht wurden von der AHA und dem ACC neue Empfehlungen zur Behandlung der Hypercholesterinämie publiziert, welche die Indikationsgrenze massiv absenken und den Einsatz einer hochdosierten Statintherapie propagieren [22]. Mehrere Validierungsstudien zeigten die grosse Gefahr der Überbehandlung durch die neuen amerikanischen Empfehlungen [23]. Die extremen Positionen des SMB und der AHA/ACC verunsichern womöglich Patienten und Ärzte. Die AGLA versucht mit ihren Empfehlungen, die Gefahren von Unter- und Überbehandlung zu vermeiden, und rät in Übereinstimmung mit den massgebenden europäischen Fachgesellschaften zu einer individuellen Risikoschätzung und einer darauf basierten, an Zielwerten orientierten Behandlung von Risikofaktoren. 


\section{Références}

1 Swiss Medical Board. Statine zur Primärprävention kardiovaskulärer Erkrankungen. Bericht vom 30.11.2013; Publikation in der Schweizerischen Ärztezeitung, Ausgabe 14 vom 2. April 2014, S. 568.

2 Brugts JJ, Yetgin T, Hoeks SE, et al. The benefits of statins in people without established cardiovascular disease but with cardiovascular risk factors: meta-analysis of randomised controlled trials. BMJ (Clinical research ed.). 2009;338:b2376.

3 Mihaylova B, Emberson J, Blackwell L, et al. The effects of lowering LDL cholesterol with statin therapy in people at low risk of vascular disease: meta-analysis of individual data from 27 randomised trials. Lancet. 2012;380:581-90.

4 Mills EJ, Rachlis B, Wu P, Devereaux PJ, Arora P, Perri D. Primary prevention of cardiovascular mortality and events with statin treatments: a network meta-analysis involving more than 65,000 patients. Journal of the American College of Cardiology. 2008;52:1769-81.

5 Ray KK, Seshasai SR, Erqou S, et al. Statins and all-cause mortality in high-risk primary prevention: a metaanalysis of 11 randomized controlled trials involving 65,229 participants. Archives of Internal Medicine. 2010;170:1024-31.

6 Taylor F, Huffman MD, Macedo AF, et al. Statins for the primary prevention of cardiovascular disease. The Cochrane Database of Systematic Reviews. 2013;1:CD004816.

7 Tonelli M, Lloyd A, Clement F, et al. Efficacy of statins for primary prevention in people at low cardiovascular risk: a meta-analysis. CMAJ: Canadian Medical Association Journal = Journal de l'Association medicale canadienne. 2011;183:E1189-1202.

8 Pletcher MJ, Lazar L, Bibbins-Domingo K, et al. Comparing impact and cost-effectiveness of primary prevention strategies for lipid-lowering. Annals of Internal Medicine. 2009;150:243-54

9 Jones PH, Davidson MH, Stein EA, et al. Comparison of the efficacy and safety of rosuvastatin versus atorvastatin, simvastatin, and pravastatin across doses (STELLAR* Trial). The American Journal of Cardiology. 2003;92:152-60.

10 Pletscher M, Plessow R, Eichler K, Wieser S. Cost-effectiveness of dabigatran for stroke prevention in atrial fibrillation in Switzerland. Swiss Medical Weekly. 2013:143:w13732.

11 Reiner Z, Catapano AL, De Backer G, et al. ESC/EAS Guidelines for the management of dyslipidaemias: the Task Force for the management of dyslipidaemias of the European Society of Cardiology (ESC) and the European Atherosclerosis Society (EAS). European Heart Journal. 2011;32:1769-818

12 Gaede P, Vedel P, Larsen N, Jensen GV, Parving HH Pedersen O. Multifactorial intervention and cardiovascular disease in patients with type 2 diabetes. The New England Journal of Medicine. 2003;348:383-93.
13 Kearney PM, Blackwell L, Collins R, et al. Efficacy of cholesterol-lowering therapy in 18,686 people with diabetes in 14 randomised trials of statins: a meta-analysis. Lancet. 2008;371:117-25.

14 De Vries FM, Denig P, Pouwels KB, Postma MJ, Hak E. Primary prevention of major cardiovascular and cerebrovascular events with statins in diabetic patients: a meta-analysis. Drugs. 2012;72:2365-73.

15 Kavousi M, Leening MJ, Nanchen D, et al. Comparison of application of the ACC/AHA guidelines, Adult Treatment Panel III guidelines, and European Society of Cardiology guidelines for cardiovascular disease prevention in a European cohort. JAMA: the Journal of the American Medical Association. 2014;311:1416-23.

16 Nanchen D, Chiolero A, Cornuz J, et al. Cardiovascular risk estimation and eligibility for statins in primary prevention comparing different strategies. The American Journal of Cardiology. 2009;103:1089-95.

17 Simpson RJ, Mendys P. The effects of adherence and persistence on clinical outcomes in patients treated with statins: A systematic review. J Clin Lipidol. 2010; $4: 462-71$.

18 Gomez-Sandoval YH, Braganza MV, Daskalopoulou SS Statin discontinuation in high-risk patients: A systematic review of the evidence. Curr Pharm Res. 2011;17:3669-89.

19 Nordestgaard BG, Chapman MJ, Humphries SE, et al Familial hypercholesterolaemia is underdiagnosed and undertreated in the general population: guidance for clinicians to prevent coronary heart disease: consensus statement of the European Atherosclerosis Society. European Heart Journal. 2013;34:3478-90a.

20 Chowdhury R, Warnakula S, Kunutsor S, et al. Association of Dietary, Circulating, and Supplement Fatty Acids With Coronary Risk: A Systematic Review and Meta-analysis. Annals of Internal Medicine. 2014;160:398406.

21 Le Boudec J, Pedrazzini B, Andrey M, Ruiz J, Cornuz J, Nanchen D. [Which nutritional advice to help reduce the cardiovascular risk? Updated of the Swiss Federal Office of Public Health's recommendations on fats consumption]. Revue médicale suisse. 2014;10:532-4, 553-8.

22 Stone NJ, Robinson J, Lichtenstein AH, et al. 2013 ACC/AHA Guideline on the Treatment of Blood Cholesterol to Reduce Atherosclerotic Cardiovascular Risk in Adults: A Report of the American College of Cardiology/American Heart Association Task Force on Practice Guidelines. Circulation. 2013.

23 Klose G, Beil FU, Dieplinger H, et al. New AHA and ACC guidelines on the treatment of blood cholesterol to reduce atherosclerotic cardiovascular risk. Wiener klinische Wochenschrift. 2014;126:169-75. 